Pacific Journal of Mathematic 


\section{INTERSECTIONS OF TERMS OF POLYCENTRAL SERIES OF FREE GROUPS AND FREE \\ LIE ALGEBRAS \\ T. C. HuRley}

This paper is concerned with deriving bases for the lower central factors of a free group modulo the intersection of certain terms of the polycentral series with the lower central series, and with the identification of the intersections themselves. The results from the group case are used to derive analogous results for the free Lie Algebra.

Let $G$ be an arbitrary group. The commutator group $[A, B]$ of the subsets $A$ and $B$ of $G$ is the subgroup generated by all commutators $[a, b]=a^{-1} b^{-1} a b, a \in A, b \in B$. The lower central series of $G$ is the chain of normal subgroups

$$
G=G_{1} \geqq G_{2} \geqq \cdots \geqq G_{i} \geqq G_{i+1} \geqq \cdots
$$

where $G_{i+1}=\left[G_{i}, G\right]$.

Let $m_{1}, \cdots, m_{n}, \cdots$ be a sequence of positive integers. For $n=$ 1 we define $G_{m_{1}}$, as above, to be the $m_{1}$ th term of the lower central series of $G$ and then define the polycentral series,

$$
G \geqq G_{m_{1}} \geqq \cdots \geqq G_{m_{1}, \cdots, m_{i}} \geqq G_{m_{1}, \cdots, m_{i}, m_{i+1}} \geqq \cdots
$$

of $G$ relative to this sequence by the rule that $G_{m_{1}, \ldots, m_{i+1}}$ is the $m_{i+1}$ th term of the lower central series of $G_{m_{1}, \ldots, m_{i}}$.

Let $L$ be a Lie Algebra. By analogy to the group case we define the polycentral series of $L$ relative to the sequence $m_{1}, \cdots, m_{i}, \cdots$

$$
L \geqq L_{m_{1}} \geqq \cdots \geqq L_{m_{1}, \cdots, m_{i}} \geqq L_{m_{1}, \cdots, m_{i}, m_{i+1}} \geqq \cdots
$$

in the following way: $L_{1}=L$ and $L_{i+1}=L_{i}$. $L$ (where in $L, A . B=$ the ideal generated by all $a . b, a \in A, b \in B$ and . denotes the multiplication in $L)$. Then, inductively, define

$$
L_{m_{1}, \cdots, m_{i+1}}=\left(L_{m_{1}, \cdots, m_{i}}\right)_{m_{i+1}} .
$$

A group $G$ is nilpotent iff $G_{n}=1_{G}$ for some $n$ and polynilpotent iff $G_{m_{1}, \cdots, m_{n}}=1_{G}$ for some (finite) sequence $m_{1}, \cdots, m_{n}$. Similarly define a nilpotent Lie Algebra and a polynilpotent Lie Algebra. The $n$th lower central factor of $G$ is $G_{n} / G_{n+1}$. A group $G$ is said to be residually- $P$, where $P$ is some group property, iff for each $g \in G$, 
$g \neq 1$, there exists a normal subgroup $N_{g}$ of $G$ such that $G / N_{g}$ has the property $P$ and $g \notin N_{g}$.

If $F$ is a free group, then $F / F_{m_{1}, \cdots, m_{n}}$ is the free polynilpotent group relative to the sequence $m_{1}, \cdots, m_{n}$. Similarly define $\mathscr{L} /$ $\mathscr{L}_{m_{1}, \ldots, m_{n}}$, the free polynilpotent Lie Algebra, where $\mathscr{L}$ is a free Lie Algebra.

Free polynilpotent groups have been studied extensively by Gruenberg [1], who shows for example that they are residually finite $p$-groups for every prime $p$, and by Smelkin [5] who determines their lower central factors and also obtains bases for the additive groups of free polynilpotent Lie Algebras. Results along these lines have also been obtained by Ward [6].

It is a consequence of Gruenberg's result that $G=F /\left(F_{m_{1}, m_{2}}, \ldots, m_{n} \cap\right.$ $\left.F_{m_{1} m_{2} \cdots m_{n}+r}\right), \quad(r \geqq 0)$, is residually nilpotent and it may also be verified that for $s \geqq m_{1} m_{2} \cdots m_{n}+r$ the $s$ th lower central factor of $G$ and of $F / F_{m_{1}, m_{2}, \cdots, m_{n}}$ are isomorphic-see Lemma 7 below. In this paper, all the lower central factors of $F /\left(F_{m, n} \cap F_{m n+r}\right)(r \geqq 0)$, are computed (Theorem $\mathrm{E}$ below). This includes Smelkin's result on $F_{m, n}$ (the case $r=0$ ) and the proof here is independent of his relying solely on computations with basic group commutators. Also a classification of $F_{m, n} \cap F_{m n+r}$ as a product of certain commutator subgroups of $F$ is obtained (Theorem A)-see Ward [6, Theorem 17.2] for the case $n=2$. The lower central factors of $F /\left(F_{m, n} \cap\right.$ $\left.F_{m n+r}\right)$ turn out to be free abelian and consequently this group is residually torsion-free-and-nilpotent and therefore by Gruenberg [1] is residually a finite $p$-group for all primes $p$.

Using the results from the group case, $\mathscr{L}_{m, n} \cap \mathscr{L}_{m n+r}$ is identified (Theorem $\left.\mathrm{A}^{\prime}\right)$ and a basis for the additive group of $\mathscr{L} /\left(\mathscr{L}_{m, n} \cap\right.$ $\mathscr{L}_{m n+r}$ ) is obtained (Theorem F).

2. Further notation and preliminary lemmas. Define, for any group $G$, with $n \geqq 2$,

$$
G(m, n ; r)=\Pi\left[G_{m+r_{1}}, G_{m+r_{2}}, \cdots, G_{m+r_{n}}\right]
$$

where the product is over all nonnegative integers $r_{1}, \cdots, r_{n}$ with $r_{1}+r_{2}+\cdots+r_{n}=r$.

If $n=1$, define $G(m, l ; r)=G_{m+r}$, (for $m \geqq 1, r \geqq 0$ ).

Then it is clear that

$$
G(m, n ; 0)=G_{m, n} .
$$

TheOREM A. $F_{m, n} \cap F_{m n+r}=F(m, n ; r)$. 
The special case

$$
F_{m, 2} \cap F_{2 m+r}=\prod_{r_{1}+r_{2}=r}\left[F_{m+r_{1}}, F_{m+r_{2}}\right]
$$

has been shown by Ward [6, Theorem 17.2].

To prove this and to describe the lower central factors of $F / F(m, n ; r)$ I need some properties of free groups, free Lie Algebras and basic commutators, and the reader is referred to either Magnus, Karrass and Solitar [4, Chapter 5] or to M. Hall [2, Chapter 11]. An excellent account may also be found in P. Hall's notes [3, Chapter 5].

For a precise formulation of bracket arrangement $\beta^{s}$ see [5, $\S 5.2]$; imprecisely $\beta^{s}\left(A_{1}, \cdots, A_{s}\right)$ means the commutator subgroup obtained by bracketing $A_{1}, \cdots, A_{s}$ in a certain fixed defined way. Thus, for example, if $\beta^{4}=((),())$ then $\beta^{4}\left(A_{1}, A_{2}, A_{3}, A_{4}\right)=\left[\left[A_{1}\right.\right.$, $\left.\left.A_{2}\right],\left[A_{3}, A_{4}\right]\right]$.

The bracket arrangement is used only in Lemma 1 below, which is probably well-known and is in any case a simple application of P. Hall's 3-subgroup lemma [5, Theorem 5.2].

Lemma 1. Let $A_{1}, \cdots, A_{s}$ be normal subgroups of a group $G$, $\beta^{s}$ a bracket arrangement of weight $s$ and $\rho$ a (fixed) permutation of $1,2, \cdots, s$. Then

$$
\beta^{s}\left(A_{\rho(1)}, A_{\rho(2)}, \cdots, A_{\rho(s)}\right) \subseteq \prod_{\sigma}\left[A_{\sigma(1)}, A_{\sigma(2)}, \cdots, A_{\sigma(s)}\right]
$$

where the product is over all permutations $\sigma$ of $1,2, \cdots, s$.

Proof. It is clear that we may assume $\rho$ is the identity permutation.

The cases $s=2$ and $s=3$ are obvious. Now use induction on $s$. Let $s>3$ and suppose $\beta^{s}=\beta^{t} \beta^{q}$ so that

$$
\beta^{s}\left(A_{1}, \cdots, A_{s}\right)=\left[\beta^{t}\left(A_{1}, \cdots, A_{t}\right), \beta^{q}\left(A_{t+1}, \cdots, A_{s}\right)\right] .
$$

By induction $\beta^{t}\left(A_{1}, \cdots, A_{t}\right) \subseteq \Pi_{\sigma}\left[A_{\sigma(1)}, \cdots, A_{\sigma(t)}\right]$ and

$$
\beta^{q}\left(A_{t+1}, \cdots, A_{s}\right) \subseteq \prod_{\rho}\left[A_{\rho(t+1)}, \cdots, A_{\rho(s)}\right]
$$

and so it is only necessary to show that

$$
\left[\left[A_{\sigma(1)}, \cdots, A_{\sigma(t)}\right],\left[A_{\rho(t+1)}, \cdots, A_{\rho(s)}\right]\right] \subseteq \prod_{\sigma}\left[A_{\sigma(1)}, \cdots, A_{\sigma(s)}\right]
$$


since for normal subgroups $[A B, C] \subseteq[A, C][B, C]$.

We can assume, since $[A, B]=[B, A]$, that $t \geqq q$, and (1) can be proved by induction on $q$. If $q=1$, then $t=s-1$ and (1) is obvious. If $q>1$ then by the 3 -subgroup lemma

$$
\begin{aligned}
& {\left[\left[A_{\sigma(1)}, \cdots, A_{\sigma(t)}\right],\left[A_{\rho(t+1)}, \cdots, A_{\rho(s)}\right]\right] } \\
& \subseteq\left[\left[A_{\sigma(1)}, \cdots, A_{\sigma(t)}, A_{\rho(s)}\right],\left[A_{\rho(t+1)}, \cdots, A_{\rho(s-1)}\right]\right] \\
& \times\left[\left[\left[A_{\sigma(1)}, \cdots, A_{\sigma(t)}\right],\left[A_{\rho(t+1)}, \cdots, A_{\rho(s-1)}\right]\right], A_{\rho(s)}\right] .
\end{aligned}
$$

Applying the inductive hypothesis to the groups on the right hand side of the above gives the required result.

$G(m, n ; r)$ as defined presents some notational difficulties and so in what follows I shall use the convention that $\Pi_{r}$ (where the expression after the product is expressed as a function of $r_{1}, \cdots, r_{n}$ ) to mean the product over all nonnegative integers $r_{1}, \cdots, r_{n}$ with $r_{1}+\cdots+r_{n}=r$.

Lemma 2. (i ) $G(m, n ; r) \subseteq G(m, n ; r-1),(r \geqq 1)$.

(ii) $[G(m, n ; r), G(m, p ; s)] \leqq G(m, n+p ; r+s)$.

(iii) $[G(m, n ; r), G(m, n ; s)] \leqq G(m, n ; r+s+1)$.

(iv) $G(m, n+1 ; r) \subseteq G(m, n ; r+1)$.

Proof. (i) is obvious.

(ii):- $[G(m, n ; r), G(m, p ; s)]=\left[\Pi_{r}\left[G_{m+r_{1}}, \cdots, G_{m+r_{n}}\right], \Pi_{s}\left[G_{m+s_{1}}\right.\right.$, $\cdots, G_{m+s}$ ]]. (If $n=1$ interpret $\Pi_{r}=G_{m+r}$ and similarly if $p=1$ ). Hence $[G(m, n ; r), G(m, p ; s)] \subseteq \Pi_{r, s}\left[\left[G_{m+r_{1}}, \cdots, G_{m+r_{n}}\right],\left[G_{m+s_{1}}, \cdots\right.\right.$, $\left.\left.G_{m+s_{p}}\right]\right]$ since for normal subgroups, $[A B, C] \subseteq[A, C][B, C]$ and $[A, B C] \subseteq[A, B][A, C]$. Now use Lemma 1 to complete the proof.

(iii):- $[G(m, n ; r), G(m, n ; s)] \leqq \Pi_{r, s}\left[\left[G_{m+r_{1}}, \cdots, G_{m+r_{n}}\right],\left[G_{m+s_{1}}, \cdots\right.\right.$, $\left.\left.G_{m+s_{n}}\right]\right]$.

If $n=1$, then $\Pi_{r, s}=\left[G_{m+r}, G_{m+s}\right]$

$$
\begin{aligned}
& \leqq G_{m+r+m+s} \cdot \\
& \subseteq G_{m+r+s+1}=G(m, n ; r+s+1) .
\end{aligned}
$$

If $n>1$

$$
\begin{aligned}
{\left[G_{m+r_{1}}, \cdots, G_{m+r_{n}}\right] } & \cong\left[G_{m+r_{1}+m+r_{2}}, G_{m+r_{3}}, \cdots, G_{m+r_{n}}\right] \\
& \cong\left[G_{m+r_{1}+r_{2}+1}, G_{m+r_{3}}, \cdots, G_{m+r_{n}}\right],
\end{aligned}
$$

and so

$$
\begin{aligned}
& {[G(m, n ; r), G(m, n ; s)]} \\
& \quad \subseteq \prod_{r, s}\left[\left[G_{m+r_{1}+r_{2}+1}, G_{m+r_{3}}, \cdots, G_{m+r_{n}}\right],\left[G_{m+s_{1}}, \cdots, G_{m+s_{n}}\right]\right] \\
& \quad \subseteq \prod_{t}\left[G_{m+t_{1}}, \cdots, G_{m+t_{2 n-1}}\right], \quad t=r+s+1 .
\end{aligned}
$$


The last step comes, as before, from Lemma 1.

(iv):- The proof is similar to (iii) and is omitted.

Lemma 3. Let $A, B, C$ be normal subgroups of the group $G$. Suppose $a \in A, b \in B, c \in C$. Then

$$
\begin{gathered}
{[a, b, c][b, c, a][c, a, b] \equiv 1 \text { modulo }[[A, B],[A, C]] .} \\
{[[A, B],[B, C]] \cdot[[A, C],[B, C]] .}
\end{gathered}
$$

Proof. By well-known commutator identities (see e.g., [4, Theorem 5.1]),

$$
\begin{aligned}
& {\left[a, b, c^{a}\right]\left[c, a, b^{c}\right]\left[b, c, a^{b}\right]=1} \\
& \quad \Longrightarrow[a, b, c[c, a]][c, a, b[b, c]][b, c, a[a, b]]=1 \\
& \quad \Longrightarrow[a, b,[c, a]][a, b, c][a, b, c,[c, a]][c, a,[b, c]] \\
& \quad \times[c, a, b][c, a, b,[b, c]][b, c,[a, b]][b, c, a][b, c, a,[a, b]]=1 .
\end{aligned}
$$

Now since

$$
\begin{aligned}
& {[a, b,[c, a]] \text { and }[a, b, c,[c, a]] \in[[A, B],[A, C]],} \\
& {[c, a,[b, c]] \text { and }[c, a, b,[b, c]] \in[[A, C],[B, C]],} \\
& {[b, c,[a, b]] \text { and }[b, c, a,[a, b]] \in[[A, B],[B, C]],}
\end{aligned}
$$

and the groups are all normal we have that

$$
\begin{gathered}
{[a, b, c][b, c, a][c, a, b] \in[[A, B],[A, C]] .} \\
{[[A, B],[B, C]] \cdot[[A, C],[B, C]] .}
\end{gathered}
$$

This lemma can be thought of as a generalization of the Jacobi identity for groups.

Corollary. Suppose $a \in G(m, n ; r), b \in G(m, p ; s), c \in G(m, q ; t)$. Then

$$
[a, b, c][b, c, a][c, a, b] \equiv 1 \text { modulo } G(m, n+p+q ; r+s+t+1) \text {. }
$$

Proof. Let $A=G(m, n ; r), B=G(m, p ; s), C=G(m, q ; t)$. Then by Lemma 2 ,

$$
\begin{aligned}
{[[A, B],[A, C]] } & \leqq[G(m, n+p ; r+s), G(m, n+q ; r+t)] \\
& \leqq G(m, n+p+q ; r+s+r+t) . \\
& \leqq G(m, n+p+q ; r+s+t+1) .
\end{aligned}
$$

Similarly for $[[A, B],[B, C]]$ and $[[A, C],[B, C]]$.

3. Bases, Let $c$ be a basic commutator with $c=\left[c_{1}, c_{2}\right]$ for 
basic commutators $c_{1}, c_{2}$. Say $c$ is structurally contained in $F(m, 1$; $r)=F_{m+r}$ iff $c \in F_{m+r}$. Suppose it is defined what is meant by saying that $c$ is structually contained in $F(m, t ; r)$ for all $1 \leqq t<n$ and for all $r$. Then say $c$ is structurally contained in $F(m, n ; r)$ iff $c_{1}$ is structurally contained in $F\left(m, s ; r_{1}\right)$, and $c_{2}$ is structurally contained in $F\left(m, t ; r_{2}\right)$ for some $s, t \geqq 1$ and some $r_{1}, r_{2} \geqq 0$ with $s+t=n$ and $r_{1}+r_{2}=r$. (Note: If $s \geqq 2$, then automatically $c_{2} \in F_{m}$ since $\left[c_{1}, c_{2}\right]$ is basic.)

For a basic commutator $c$, write $c \bar{\epsilon} F(m, n ; r)$ iff $c$ is structurally contained in $F(m, n ; r)$.

The following lemmas are easily verified.

LEMMA 4. If $c \bar{\in} F(m, n ; r)$ then $c \in F(m, n ; r)$.

Lemma 5. (i ) If $c \bar{\in} F(m, n ; r)$ then (ii) $c \bar{\in} F(m, n-1 ; r+m)$, $(n \geqq 2) . \quad c \bar{\in} F(m, n ; r-1) . \quad(r \geqq 1)$.

Proofs. These come directly from the definition and Lemma 2.

LemMa 6. Suppose $b, c$ are basic commutators such that $b \bar{\epsilon}$ $F(m, s ; i)$, and $c \bar{\in} F(m, t ; k)$, with $s+t=n$ and $i+k=r$. Then $[b, c]$ is congruent, modulo $F(m, n ; r+1)$, to a product of basic commutators, each of which is structurally contained in $F(m, n ; r)$.

Proof. We may assume that $F$ is finitely generated since $b$ and $c$ only involve a finite number of generators of $F$.

We may also assume that $b>c$ since $[b, c]=[c, b]^{-1}$. If $b$ is a generator of $F$ then clearly $[b, c]$ is a basic commutator. If $b=$ $\left[b_{1}, b_{2}\right]$ where $b_{2} \leqq c$ then again $[b, c]$ is a basic commutator and there is nothing to be shown.

Define the difference number (d.n.) of a commutator $[b, c]$ where $b$ and $c$ are basic commutators with $b>c$, in the following way: Let $b$ have weight $w$ and suppose there exists $v$ basic commutators of weight $\leqq w$. Let $c$ be the $l$ th basic commutator. Then $l<v$ and define

$$
\text { d.n. }[b, c]=v-l \text {. }
$$

Now use induction on d.n. $[b, c]$. (This is a type of "backward induction").

Suppose $b=\left[b_{1}, b_{2}\right]$ where $b_{1}, b_{2}$ are basics and $b_{2}>c$. Then weight $b_{2} \geqq$ weight $c$, giving that $b_{2} \in F_{m}$ and consequently $b_{1} \in F_{m}$. 
Thus, we may assume $s \geqq 2$ and therefore suppose

$$
b_{1} \bar{\epsilon} F\left(m, s_{1} ; i_{1}\right), \quad b_{2} \bar{\epsilon} F\left(m, s_{2} ; i_{2}\right),
$$

with $s_{1}+s_{2}=s, i_{1}+i_{2}=i,\left(s_{1}, s_{2} \geqq 1, i_{1}, i_{2} \geqq 0\right)$. Then by corollary to Lemma 3 ,

(3) $[b, c]=\left[b_{1}, b_{2}, c\right] \equiv\left[b_{1}, c, b_{2}\right]\left[b_{2}, c, b_{1}\right]^{-1}$ modulo $F(m, n ; r+1)$.

Now d.n. $\left[b_{1}, c\right]<$ d.n. $[b, c]$ and so by the inductive hypothesis $\left[b_{1}, c\right]$ is congruent, modulo $F\left(m, s_{1}+t ; i_{1}+k+1\right)$, to a product, $e_{1}^{\alpha_{1}} \cdots e_{q}^{\alpha_{q}}$ say $\left(\alpha_{i} \in Z\right)$, of basic commutators $e_{i}$ with each $e_{i} \bar{\epsilon} F\left(m, s_{1}+\right.$ $\left.t ; i_{1}+k\right)$.

Then using the commutator identity $[x y, z]=[x, z][x, z, y][y, z]$ and Lemmas 2 and 3 , we see that

$$
\left[b_{1}, c, b_{2}\right]=\prod_{j=1}^{q}\left[e_{j}, b_{2}\right]^{\alpha_{j}} \text { modulo } F(m, n ; r+1) \text {. }
$$

Now for each $j, 1 \leqq j \leqq q$, weight $e_{j} \leqq$ weight $b$. Also $b_{2}>c$ and hence d.n. $\left[e_{j}, b_{2}\right]<$ d.n. $[b, c]$. Therefore by induction each $\left[e_{j}, b_{2}\right]$, and consequently $\left[b_{1}, c, b_{2}\right]$, can be written, modulo $F(m, n$; $r+1)$, as a product of basic commutators structurally contained in $F(m, n ; r)$.

Also d.n. $\left[b_{2}, c\right]<$ d.n. $[b, c]$ and therefore $\left[b_{2}, c\right]$ is congruent, modulo $F\left(m, s_{2}+t ; i_{2}+k+1\right)$, to a product, $f_{1}^{\beta_{1}} \cdots f_{h}^{\beta}$ say $\left(\beta_{i} \in \boldsymbol{Z}\right)$, of basic commutators $f_{i}$ such that $f_{i} \bar{\epsilon} F\left(m, s_{2}+t ; i_{2}+k\right)$.

For a particular $f_{i}$, note that there are two possibilities, either $f_{i} \geqq b_{1}$ or $f_{i}<b_{1}$. In any case, as above, get

$$
\left[b_{2}, c, b_{1}\right] \equiv \prod_{j=1}^{h}\left[f_{j}, b_{1}\right]^{\beta_{j}} \text { modulo } F(m, n ; r+1) \text {. }
$$

Now consider the two cases.

Case 1. $f_{j} \geqq b_{1}$. Now weight $f_{j} \leqq$ weight $b$ and also $b_{1}>b_{2}>c$ giving that d.n. $\left[f_{j}, b_{1}\right]<$ d.n. $[b, c]$.

Case 2. $b_{1}>f_{j}$. Now weight $b_{1}<$ weight $b$ and also, since $f_{j}$ has bigger weight than $c, f_{j}>c$ giving that d.n. $\left[b_{1}, f_{j}\right]<$ d.n. $[b, c]$.

Hence, by induction, each $\left[f_{j}, b_{1}\right]$ is congruent, modulo $F(m, n$; $r+1)$, to a product of basic commutators structurally contained in $F(m, n ; r)$ and therefore from (5), so is $\left[b_{2}, c, b_{1}\right]$. Thus $\left[b_{1}, c, b_{2}\right]$ and $\left[b_{2}, c, b_{1}\right]^{-1}$ are products, modulo $F(m, n ; r+1)$, of basic commutators of the required form and using (3) gives the required result.

THEOREM B. $F(m, n ; r) / F(m, n ; r+1)$ is free abelian, freely 
generated by those basic commutators $c$ of weight $m n+r$ such that $c \bar{\epsilon} F(m, n ; r)$.

Proof. It follows from Lemma 2 that the group is abelian. Also since $F(m, n ; r) \subseteq F_{m n+r}$, and $F(m, n ; r+1) \subseteq F_{m n+r+1}$ the freeness will follow from the Basis Theorem for Basic Commutators [4, Theorem 5.13A or 2, Theorem 11.2.4].

Therefore it is only necessary to show that the stated basic commutators generate the group.

If $n=1$, this is equivalent to showing that $F_{m+r} / F_{m+r+1}$ is generated by basic commutators of weight $m+r$ and this is part of the Basis Theorem. Let $n>1$ and proceed by induction on $n$. If $a \in F(m, n ; r)$ then $a$ is a product of elements of the form

$$
b=\left[b_{1}, \cdots, b_{n}\right], b_{i} \in F_{m+r_{i}} \text { with } r_{1}+\cdots+r_{n}=r .
$$

By induction $\left[b_{1}, \cdots, b_{n-1}\right]$ is congruent, modulo $F\left(m, n-1 ; r-r_{n}+1\right)$ to a product of basic commutators structurally contained in $F\left(m, n-1 ; r-r_{n}\right)$ and $b_{n}$ is also congruent modulo $F_{m+r_{n}+1}$ to a product of basic commutators contained in $F_{m+r_{n}}$. Now from Lemma 2,

$$
\left[F\left(m, n-1 ; r-r_{n}+1\right), F_{m+r_{n}}\right] \subseteq F(m, n ; r+1)
$$

and

$$
\left[F\left(m, n-1 ; r-r_{n}\right), F_{m+r_{n}+1}\right] \subseteq F(m, n ; r+1)
$$

and so the problem reduces to showing that $[a, b]$, with $a \bar{\epsilon} F(m, n-$ $\left.1 ; r-r_{n}\right)$ and $b \bar{\epsilon} F\left(m, 1 ; r_{n}\right)$, is congruent, modulo $F(m, n ; r+1)$, to a product of basic commutators of weight $m n+r$ each of which is structurally contained in $F(m, n ; r)$. Lemma 6 does this for us.

Corollary. Let $b$ be a basic commutator. Then

$$
b \bar{\epsilon} F(m, n ; r) \Longleftrightarrow b \in F(m, n ; r) \text {. }
$$

Proof. We have already seen (Lemma 4) that if $b \bar{\epsilon} F(m, n ; r)$ then $b \in F(m, n ; r)$.

Suppose now $b \in F(m, n ; r)$. Then $b$, being a basic commutator, is not 1 and so $b \notin \bigcap_{i=1}^{\infty} F_{i}$, since free groups are residually nilpotent. Thus $b \notin \bigcap_{i=1}^{\infty} F(m, n ; i)$ and suppose $b \in F(m, n ; s), b \notin F(m, n ; s+1)$. So $\mathrm{s} \geqq r$ and therefore by Theorem $\mathrm{B}, b$ is a unique product, modulo $F(m, n ; s+1)$ of basic commutators structually contained in $F(m, n ; s)$. Therefore since $b$ is also a basic commutator, this unique product must be precisely $b$, giving that $b \bar{\in} F(m, n ; s)$ and therfore $b \bar{\epsilon}(m, n ; r)$. 
THEOREM C. Every element of $F_{m, n}$ can be written uniquely as

$$
c^{\alpha_{1}} \cdots c^{\alpha_{s}} \bmod F(m, n ; r),
$$

where $c_{1}, \cdots, c_{s}$ are the basic commutators of weight $<m n+r$ structually contained in $F_{m, n}, \alpha_{i} \in Z$, and $c_{1}<\cdots<c_{s}$ in the ordering of the basic commutators.

REMARK. This theorem reduces to P. Hall's Basis Theorem for the case $n=1$ and $m=1$. This then can be considered as a "basis theorem" for certain terms of the polycentral series of $F$. Whereas in the Basis Theorem uniqueness modulo $F_{r+1}$ is obtained, here $F_{r+1}$ must be replaced by the complicated expression $F(m, n ; r)$.

Proof of Theorem $C$. The proof is immediate from Theorem B. I omit spelling out the details.

\section{THEOREM A.}

$$
F_{m, n} \cap F_{m n+r}=F(m, n ; r)
$$

Proof. The proof is now easy. Use induction on $r$. The case $r=0$ is trivial.

It is clear that $F(m, n ; r) \subseteq F_{m, n} \cap F_{m n+r}$. Suppose $r>0$ and $a \in F_{m, n} \cap F_{m n+r}$. Then $a \in F(m, n ; r-1)$ by the inductive hypothesis, and by Theorem $\mathrm{B}, a$ is a product, modulo $F(m, n ; r)$, of certain basic commutators of weight $m n+r-1$. Since also $a \in F_{m n+r}$, this product must be 1 by the Basis Theorem, and so $a \in F(m, n ; r)$. This proves Theorem A.

It is clear that these arguments work for the free Lie Algebra and so I state without proof the following two theorems.

Let $T_{i}$ denote the set of basic commutators of weight $i$.

THEOREM B'. $\mathscr{L}_{m, n} / \mathscr{L}(m, n ; r)$ has as additive basis those basic commutators of weight $<m n+r$ which are structually contained in $\mathscr{L}_{m, n}$.

THEOREM $\mathrm{A}^{\prime}$.

$$
\mathscr{L}_{m, n} \cap \mathscr{L}_{m n+r}=\mathscr{L}(m, n ; r) \text {. }
$$

Theorems $\mathrm{A}^{\prime}$ and $\mathrm{B}^{\prime}$ may also be deduced easily from Theorem $\mathrm{F}$ and its corollary below. 
THEOREM D. (Smelkin [5]). Let $G=F / F_{m, n}$. Then $G_{i} / G_{i+1}$ is free abelian, freely generated by the set $S_{i}=T_{i} \backslash R_{i}$, where $R_{i}$ denotes the basic commutators of weight $i$ structurally contained in $F_{m, n}$.

Proof. Clearly $G_{i} / G_{i+1}$ is generated by $S_{i}$ since the basics structurally contained in $F_{m, n}$ vanish in $G$.

We need only consider $i \geqq m n$ and hence suppose $i=m n+r$, $r \geqq 0$.

Suppose a product $\Pi$ of elements from $S_{i}$ is in $F_{i+1} F_{m, n}$. Then

$$
\Pi=a b, \quad a \in F_{m, n}, \quad b \in F_{i+1} .
$$

Hence $a \in F_{m, n} \cap F_{i}=F(m, n ; r)$ by Theorem A. Therefore by Theorem B, $a$ is a product, modulo $F(m, n ; r+1)\left(\subseteq F_{i+1}\right)$, of basic commutators of weight $i$ which are structurally contained in $F(m, n ; r)$. The set of basic commutators structually contained in $F(m, n ; r)$ of weight $i$ has empty intersection with $S_{i}$ and so $a \equiv 1$ modulo $F_{i+1}$ and hence $\Pi \equiv 1$ modulo $F_{i+1}$, giving that $\Pi$ is identically 1.

Theorem D'. (Smelkin [5]). The set consisting of those basic commutators which are not structurally contained in $\mathscr{L}_{m, n}$ is an additive basis for $\mathscr{L} \mid \mathscr{L}_{m, n}$.

This may be proved from Theorem $\mathrm{A}^{\prime}$ by analogy to the group case. It is also a trivial consequence of Theorem $\mathrm{F}$ below.

The proof of the following theorem includes that of Theorem D.

THEOREM E. Let $G=F / F(m, n ; r)$. Then $G_{i} / G_{i+1}$ is free abelian, freely generated by the set $S_{i}=T_{i} \backslash R_{i}$, where $R_{i}$ denotes the basic commutators of weight $i$ structurally contained in $F(m, n ; r)$.

Proof. Clearly $S_{i}$ generates $G_{i} / G_{i+1}$. It is only necessary to consider $i \geqq m n+r$ and hence let $i=m n+r+s, s \geqq 0$. Suppose a product $\Pi$ of elements from $S_{i}$ is contained in $F(m, n ; r) F_{i+1}$.

Then

$$
\Pi=a b, a \in F(m, n ; r), \quad b \in F_{i+1} \text {. }
$$

Therefore $a \in F_{i} \cap F(m, n ; r)=F(m, n ; r+s)$, by Theorem A. Thus $a$, by Theorem $\mathrm{B}$, is a product, modulo $F(m, n ; r+s+1)$, of basic commutators of weight $i$ which are structurally contained in 
$F(m, n ; r+s)$. An element of $S_{i}$ cannot be structurally contained in $F(m, n: r+s)$ for if so it would be constructurally contained in $F(m, n ; r)$-see Lemma 5 . Hence $\Pi$ is identically 1 .

If $G$ is any group then as e.g., in Magnus, Karrass and Solitar [4, Chapter 5] $\bigoplus_{i=1}^{\infty} G_{i} / G_{i+1}$ can be made into a Lie Algebra $L$ by defining addition, + , in $L$ by

$$
\sum a_{i} G_{i+1}+\sum a_{i}^{\prime} G_{i+1}=\sum a_{i} a_{i}^{\prime} G_{i+1},\left(a_{i}, a_{i}^{\prime} \in G_{i}\right)
$$

(i.e., using the group multiplication componentwise) and multiplication, ', in $L$ by first defining

$$
a_{i} G_{i+1} \cdot a_{j} G_{j+1}=\left[a_{i}, a_{j}\right] G_{i+j+1},\left(a_{i} \in G_{i}, a_{j} \in G_{j}\right)
$$

and then using the distributive laws to extend this definition to all of $\oplus G_{i} / G_{i+1}$.

Let $G=F / F(m, m ; r)$. Then there is the obvious surjective Lie Algebra homomorphism $\sigma: \mathscr{L} / \mathscr{L}(m, n ; r) \rightarrow \bigoplus G_{i} / G_{i+1}$. The basic commutators structurally contained in $\mathscr{L}(m, n ; r)$ vanish in $\mathscr{L} / \mathscr{L}(m, n ; r)$ and the others are linearly independent in $\oplus G_{i} / G_{i+1}$ by Theorem $\mathrm{E}$. This gives the following theorem.

THEOREM F. The additive group of $\mathscr{L} / \mathscr{L}(m, n ; r)$ has as basis the set of those basic commutators which are not structurally contained in $\mathscr{L}(m, n ; r)$.

Corollary. $\mathscr{L} / \mathscr{L}(m, n ; r)$ and $\oplus G_{i} / G_{i+1}$ are isomorphic Lie Algebras under the obvious map.

Lemma 7. (i) If $F / R$ is residually nilpotent so is $F /\left(R \cap F_{n}\right)$.

(ii) The mth lower central factor of $F / R$ is isomorphic to that of $F /\left(R \cap F_{n}\right)$ for any $m \geqq n$.

Proof. (i) If $F / R$ is residually nilpotent, then $\bigcap_{i} F_{i} R=R$. Now $\bigcap_{i} F_{i}\left(R \cap F_{n}\right) \subseteq \bigcap_{i} F_{i} R=R$. Also for $i \geqq n, F_{i}\left(R \cap F_{n}\right) \subseteq F_{n}$ giving that $\bigcap_{i} F_{i}\left(R \cap F_{n}\right)=R \cap F_{n}$. Thus $F /\left(R \cap F_{n}\right)$ is residually nilpotent when $F / R$ is.

(ii) Since $F_{m+1}\left(R \cap F_{n}\right) \subseteq F_{m+1} R$ we have a surjective map

$$
F_{m}\left(R \cap F_{n}\right) / F_{m+1}\left(R \cap F_{n}\right) \longrightarrow F_{m} R / F_{m+1} R \text {. }
$$

The kernel of this map is $F_{m}\left(R \cap F_{n}\right) \cap F_{m+1} R=F_{m+1}\left(R \cap F_{n}\right)$, for $m \geqq n$, giving that the two groups are isomorphic.

CoRollaRY 1. $F /\left(F_{m_{1}, \cdots, m_{n}} \cap F_{m_{1} \cdots m_{n}+r}\right)$ is residually nilpotent. 
COROLLARY 2. $F /\left(F_{m, n} \cap F_{m n+r}\right)$ is residually torsion-free and nilpotent.

CoROLlaRY 3. $F /\left(F_{m, n} \cap F_{m n+r}\right)$ is residually a finite p-group for all primes $p$.

Proof of corollaries. By Gruenberg [1], $F / F_{m_{1}, \ldots, m_{n}}$ is residually nilpotent, giving that $F /\left(F_{m_{1}, \cdots, m_{n}} \cap F_{m_{1} \cdots m_{n}+r}\right)$ is residually nilpotent. By Theorem E, $F /\left(F_{m, n} \cap F_{m n+r}\right)$ has torsion-free lower central factors and is therefore residually torsion-free-and-nilpotent and hence again by Gruenberg [1] it is residually a finite $p$-group for all primes $p$.

It is also a corollary that the sth lower central factor of $F / F_{m_{1}, \cdots, m_{n}}$ is isomorphic to that of $F /\left(F_{m_{1}, \cdots, m_{n}} \cap F_{m_{1} \cdots m_{n}+r}\right)$, for $s \geqq$ $m_{1} \cdots m_{n}+r$.

\section{REFERENCES}

1. K. W. Gruenberg, Residual properties of infinite soluble groups, Proc. London Math. Soc., (3) 7 (1957), 29-62.

2. M. Hall, The Theory of Groups, Macmillan, New York, 1959.

3. - The Edmonton Notes on Nilpotent Groups, Queen Mary College Lecture Notes, London, 1969.

4. W. Magnus, A. Karrass and D. Solitar, Combinatorial Group Theory, Interscience, New York-London-Sydney, 1966.

5. A. L. Smelkin, Free Polynilpotent Groups, Izv. Akad. Nauk SSSR Ser. Mat., 28 (1964), 91-122; English transl., Amer. Math. Soc. Transl., (2) 55 (1966), 270-304.

6. M. A. Ward, Basic Commutators, Phil. Trans. Royal Soc. of London, Ser, A, 264 (1969), 343-412.

Received September 13, 1977 and in revised form June 9, 1978.

UNIVERSITY COLLEGE

Belfield, Dublin 4, Ireland 


\section{PACIFIC JOURNAL OF MATHEMATICS}

\section{EDITORS}

DoNALD BABBITT (Managing Editor)

University of California

Los Angeles, California 90024

HUGo Rossi

University of Utah

Salt Lake City, UT 84112

C. C. MOORE and ANDREW OGG

University of California

Berkeley, CA 94720
J. DUGUNDJI

Department of Mathematics University of Southern Californı Los Angeles, California 90007

R. Finn and J. Milgram Stanford University Stanford, California 94305

\section{ASSOCIATE EDITORS}

E. F. BECKENBACH

B. H. Neumann
F. WOLF

K. YOSHIDA

\section{SUPPORTING INSTITUTIONS}

UNIVERSITY OF BRITISH COLUMBIA CALIFORNIA INSTITUTE OF TECHNOLOGY UNIVERSITY OF CALIFORNIA MONTANA STATE UNIVERSITY UNIVERSITY OF NEVADA, RENO NEW MEXICO STATE UNIVERSITY OREGON STATE UNIVERSITY UNIVERSITY OF OREGON
UNIVERSITY OF SOUTHERN CALIFORNIA STANFORD UNIVERSITY UNIVERSITY OF HAWAII UNIVERSITY OF TOKYO UNIVERSITY OF UTAH WASHINGTON STATE UNIVERSITY UNIVERSITY OF WASHINGTON 


\section{Pacific Journal of Mathematics}

Vol. 82, No. $1 \quad$ January, 1979

Werner Bäni, Subspaces of positive definite inner product spaces of countable dimension ...................................... 1

Marilyn Breen, The dimension of the kernel of a planar set..............

Kenneth Alfred Byrd, Right self-injective rings whose essential right ideals

are two-sided

Patrick Cousot and Radhia Cousot, Constructive versions of Tarski's fixed

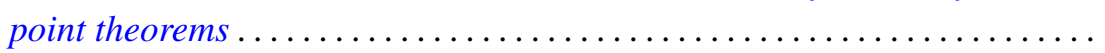

Ralph S. Freese, William A. Lampe and Walter Fuller Taylor, Congruence lattices of algebras of fixed similarity type. $I \ldots \ldots \ldots \ldots \ldots \ldots \ldots$

Cameron Gordon and Richard A. Litherland, On a theorem of Murasugi .....

Mauricio A. Gutiérrez, Concordance and homotopy. I. Fundamental

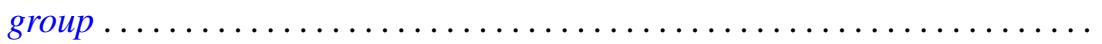

Richard I. Hartley, Metabelian representations of knot groups .............

Ted Hurley, Intersections of terms of polycentral series of free groups and free

Lie algebras ........................................

Roy Andrew Johnson, Some relationships between measures ............ 117

Oldřich Kowalski, On unitary automorphisms of solvable Lie algebras .......

Kee Yuen Lam, $K O$-equivalences and existence of nonsingular bilinear

maps...................................................

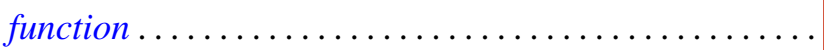

Robert A. Messer and Alden H. Wright, Embedding open 3-manifolds in compact 3-manifolds ............................

Gerald Ira Myerson, A combinatorial problem in finite fields. I . .

James Nelson, Jr. and Mohan S. Putcha, Word equations in a band of paths.

Baburao Govindrao Pachpatte and S. M. Singare, Discrete generalized Gronwall inequalities in three independent variables . .

William Lindall Paschke and Norberto Salinas, $C^{*}$-algebras associated with free products of groups ........................

Bruce Reznick, Banach spaces with polynomial norms ....

David Rusin, What is the probability that two elements of a finite group

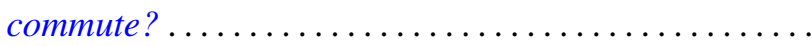

M. Shafii-Mousavi and Zbigniew Zielezny, On hypoelliptic differential operators of constant strength ...

Joseph Gail Stampfli, On selfadjoint derivation ranges .... . . .

Robert Charles Thompson, The case of equality in the matrix-valued triangle

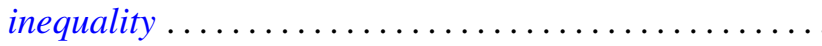

Marie Angela Vitulli, The obstruction of the formal moduli space in the negatively graded case. 